\title{
Dachverbände als Instrument der Non-Profit Governance ${ }^{*}$
}

\author{
GEORG VON SCHNURBEIN ${ }^{* *}$
}

Die Forschung zu Non-Profit Governance bezieht sich dominant auf den Vorstand einer Non-Profit-Organisation (NPO). Dabei spielen weit mehr Akteure eine Rolle für die Governance einer NPO. Dieser Beitrag befasst sich auf der Grundlage des Institutionellen Isomorphismus mit der Bedeutung des Dachverbandes als GovernanceInstrument der Mitgliederorganisationen. In Abhängigkeit von der Autonomie der Mitgliederorganisationen und der Umfeldstabilität wird idealtypisch aufgezeigt, wie die Mitgliedschaft im Dachverband Anpassungsprozesse fördert und welche Entwicklungspfade dadurch ermöglicht werden.

Schlagwörter: Dachverbände, Isomorphismus, Mitglieder, Non-Profit Governance, Reputation, Stakeholder

\section{Umbrella Associations as Governance Instrument of Nonprofit Organizations}

Research on nonprofit governance is dominantly focused on the board, although other constituents play important roles, as well. This piece of work uses the framework of institutional isomorphism to discuss the role of an umbrella foundation as a governance instrument for its member organizations. Based on the autonomy of the members and the stability of the environment as decisive factors, four ideal type influences of the umbrella organization on changes in the governance system are presented.

Keywords: Umbrella Associations, Isomorphism, Members, Nonprofit Governance, Reputation, Stakeholder

\section{1. $\quad$ Einleitung}

Dachverbände zählen zu jenen eher seltenen Typen von Non-Profit-Organisationen (NPO), deren spezifische Eigenschaften oftmals übersehen werden (Young 2001). Dabei spielen Dachverbände im gesellschaftlichen Gesamtgefüge eine nicht unerhebliche Rolle. Sie sind als Vertreter von Wirtschaftsbranchen oder anderen Gesellschaftsgruppen an der politischen Willensbildung beteiligt und tragen wesentlich zur Organisation des Non-Profit-Sektors bei. Ebenso sind sie ein Merkmal föderalistischer Staatssysteme, in denen Non-Profit-Organisationen oftmals die geographische

Beitrag eingereicht am 31.10.2011; nach doppelt verdecktem Gutachterverfahren überarbeitete Fassung angenommen am 3.2.2012.

** Prof. Dr. Georg von Schnurbein, Centre for Philanthropy Studies (CEPS) Universität Basel, Peter Merian-Weg 6, CH-4051 Basel, Tel.: +41 6126734 63, Fax: +41 61267 23 93, E-Mail: georg.vonschnurbein@unibas.ch, Forschungsschwerpunkte: Stiftungsmanagement, Non-Profit Governance, Non-Profit Management. 
Struktur des Landes nachbilden. Auch wenn Dachverbände als Vereine selbst NPO sind, liegt der Fokus dieses Beitrags auf dem Nutzen von Dachverbänden als Governance-Instrument anderer NPO, vornehmlich der Mitglieder des Dachverbandes.

Non-Profit Governance wird in diesem Beitrag verstanden als „ein Set von Instrumenten, Maßnahmen und Mechanismen, das den Vorstand einer NPO bei der Globalsteuerung und der wirksamen Wahrnehmung seiner Aufgaben unterstützt sowie die Zweckerfüllung, Legitimität und Verantwortlichkeit der Organisation bzw. die Berücksichtigung der Stakeholderinteressen sicherstellt" (von Schnurbein 2008: 86). Dieses weit gefasste Begriffsverständnis hebt insbesondere die Einbindung verschiedener Stakeholder in die strategische Steuerung der Organisation hervor.

Durch die Auseinandersetzung mit Dachverbänden als Governance-Instrument wird ein vernachlässigtes Thema zur Non-Profit Governance aufgegriffen. Denn obwohl inzwischen eine Vielzahl von Theorieansätzen besteht, dominiert in der wissenschaftlichen Auseinandersetzung zur Non-Profit Governance nach wie vor die Prinzipal-Agenten-Theorie (Cornforth 2011). Dies hat zur Folge, dass der konzeptionelle Akzent dominant auf dem Vorstand und dessen Beziehung zur Geschäftsführung liegt. Zur Zusammensetzung, Arbeitweise und Leistungsorientierung des Vorstands finden sich in der Literatur inzwischen vielfältige theoretische und empirische Lösungsansätze und Ergebnisse (Brown/Iverson 2004). Die Mehrzahl der empirischen Untersuchungen zur Non-Profit Governance beruht auf der Befragung von Vorstand oder Geschäftsführung. Einschätzungen und Meinungen anderer Beteiligter werden kaum in die Analysen mit einbezogen. Dabei wird die Bedeutung der Stakeholder für eine effektive Governance aus theoretischer Sicht nicht in Zweifel gezogen. Vereinzelte Beiträge zur Bedeutung von Mitgliedern (Graf 2007; von Schnurbein 2009) oder anderen Stakeholdern (Bradshaw 2007; Balser/McClusky 1995; Saidel 1998) belegen diesen Befund.

Dieser Beitrag setzt sich mit der Bedeutung von Dachverbänden als GovernanceAkteure anderer NPO, insbesondere seiner Mitgliederorganisationen auseinander. Die Fragestellung lautet daher: In welcher Form kann ein Dachverband zum GovernanceSystem seiner Mitgliederorganisationen beitragen?

Dazu werden zunächst das Begriffsverständnis sowie die unterschiedlichen Formen von Dachverbänden diskutiert. Anschließend wird der Institutionelle Isomorphismus als theoretische Grundlage der nachfolgenden Diskussion vorgestellt. Unter Berücksichtigung von Mitgliederautonomie und Umweltsituation wird ein Modell entwickelt, das durch den Dachverband bewirkte isomorphe Prozesse in der Governance der Mitgliederorganisationen differenziert. Darauf aufbauend werden Hypothesen zu Entwicklungsprozessen innerhalb des Modells gebildet.

\section{Dachverbände als besondere NPO-Form}

Die Bezeichnung Dachverband für eine Organisation ist kein rechtlich definierter Begriff und kann daher weitgehend frei verwendet werden. Auch lässt sich aus dem Namen eines Verbandes nicht ausdrücklich erkennen, ob es sich dabei um einen Dachverband handelt. Als Verständnisgrundlage sollen daher im weiteren Verlauf die folgenden Kriterien gelten: 
- Ein Dachverband hat eine formalrechtliche Struktur, i. d. R. als Verein. Im Gegensatz zu Netzwerken und Initiativen ist ein Dachverband eine eigenständige Rechtsperson.

- Der Zusammenschluss in einem Dachverband hat nationalen oder internationalen Charakter.

- Die Mitglieder verbindet eine geographische oder thematisch-inhaltliche Zusammengehörigkeit.

- Die Mitglieder eines Dachverbandes sind selbst Verbände, d. h. eigenständige Vereine mit Mitgliedern. Die Mitglieder dieser Subverbände können sowohl Kollektivmitglieder (Vereine) oder Einzelmitglieder sein.

- Die Mitglieder sind i. d. R. rechtlich unabhängig vom Dachverband. Dieses letzte Kriterium trifft jedoch nicht auf alle Dachorganisationen zu.

Die Strukturen eines Dachverbandes sind häufig das Ergebnis einer historischen Entwicklung, und heutige Dachverbände stellen teilweise das Ergebnis aus der Fusion mehrerer Dachverbände dar (Schwarz 2005). Bradshaw (2007) hebt hervor, dass in solchen Strukturen nie vollständige Stabilität, Klarheit oder Konsens erreicht wird. Young et al. (1996) unterscheiden auf der Grundlage einer empirischen Untersuchung mit 183 Dachverbänden drei Strukturformen im Aufbau von Dachverbänden, deren Beschreibung mit Vor- und Nachteilen in Tabelle 1 zusammengefasst werden. Die Unterscheidung stellt drei Grundtypen dar, die in der Realität nicht trennscharf zu differenzieren sind.

\begin{tabular}{|c|c|c|c|}
\hline & Ausprägungen & Vorteile & Nachteile \\
\hline $\begin{array}{l}\text { föderalis- } \\
\text { tische } \\
\text { Struktur }\end{array}$ & $\begin{array}{l}\text { Eine hierarchische Struk- } \\
\text { tur mit autonomen Or- } \\
\text { ganisationen; die natio- } \\
\text { nale Organisation be- } \\
\text { stimmt die Verbandspo- } \\
\text { litik, erfüllt die nationale } \\
\text { Interessenvertretung und } \\
\text { bietet Dienste für die } \\
\text { föderalen Organisatio- } \\
\text { nen an. }\end{array}$ & $\begin{array}{l}\text { - Hohe Kontrolle der } \\
\text { zentralen Führung } \\
\text { durch die Mitglieder } \\
\text { - Verbandspolitik } \\
\text { wird durch Zentrale } \\
\text { festgelegt } \\
\text { - Gemeinsames Auf- } \\
\text { treten }\end{array}$ & $\begin{array}{l}\text { - Geringe Durchset- } \\
\text { zungsfähigkeit der } \\
\text { Zentrale } \\
\text { - Konkurrenzsitua- } \\
\text { tion zwischen } \\
\text { Zentrale und de- } \\
\text { zentralen Einhei- } \\
\text { ten (z. B. Fundrai- } \\
\text { sing) }\end{array}$ \\
\hline $\begin{array}{l}\text { Verbund- } \\
\text { struktur }\end{array}$ & $\begin{array}{l}\text { Zusammenschluss von } \\
\text { mehreren, autonomen } \\
\text { Organisationen aus dem } \\
\text { gleichen Tätigkeitsgebiet, } \\
\text { die einer nationalen Or- } \\
\text { ganisation untergeordnet } \\
\text { sind, die ihre Interessen } \\
\text { vertritt und ihnen Diens- } \\
\text { te anbietet. }\end{array}$ & $\begin{array}{l}\text { - Hohe Kontrolle der } \\
\text { zentralen Führung } \\
\text { durch die Mitglieder } \\
\text { - Verbandspolitik } \\
\text { wird von dezentra- } \\
\text { len Einheiten selbst } \\
\text { entschieden } \\
\text { - Größere Unabhän- } \\
\text { gigkeit der dezentra- } \\
\text { len Einheiten }\end{array}$ & $\begin{array}{l}\text { - Kaum Durchset- } \\
\text { zungsfähigkeit der } \\
\text { Zentrale } \\
\text { - Uneinheitliche } \\
\text { Erscheinung und } \\
\text { Politik }\end{array}$ \\
\hline
\end{tabular}




\begin{tabular}{|c|c|c|c|}
\hline $\begin{array}{l}\text { Konzern- } \\
\text { struktur }\end{array}$ & $\begin{array}{l}\text { Eine zentralistische Or- } \\
\text { ganisation mit lokalen } \\
\text { Einheiten, die von der } \\
\text { nationalen Organisation } \\
\text { gesteuert und kontrol- } \\
\text { liert werden. }\end{array}$ & $\begin{array}{l}\text { - Standards können } \\
\text { durch die zentrale } \\
\text { Kontrolle überall } \\
\text { gleich gewährleistet } \\
\text { werden } \\
\text { - Einheitliches Auftre- } \\
\text { ten (Franchise) } \\
\text { - Verantwortung liegt } \\
\text { bei der Zentrale }\end{array}$ & $\begin{array}{l}\text { - Kaum eigene Ge- } \\
\text { staltungsmöglichke } \\
\text { iten für dezentrale } \\
\text { Einheiten }\end{array}$ \\
\hline
\end{tabular}

Tabelle 1: Unterschiedliche Strukturen in Dachverbänden (eigene Darstellung in Anlebnung an Young et al. 1996)

Eine häufige Gestaltung von Dachverbänden ist die föderalistische Struktur, die dem föderalistischen Staatsaufbau entlehnt ist. Hier besteht eine klare Aufgabentrennung zwischen der nationalen Zentrale und den dezentralen Einheiten sowie eine hierarchische (meist geographisch gestaltete) Ordnung. In Deutschland wird die hierarchische Struktur auch in der Bezeichnung deutlich: Der TSV 1866 München ist Mitglied im Bayrischen Fußballverband, der wiederum Mitglied im Deutschen Fußballbund ist. Schließen sich in einem Dachverband mehrere themenverwandte Verbände zusammen, dann entwickelt sich eher eine Verbundstruktur. Die Mitglieder werden durch die Dachorganisation kaum in ihrer Autonomie eingeschränkt, vielfach entspricht der Verbandszweck dem kleinsten gemeinsamen Nenner der Mitgliederverbände. Schließlich gibt es noch die Konzernstruktur, die häufig eine rechtliche Abhängigkeit der regionalen oder fachlichen Einheiten von der Zentrale beinhaltet (Oster 1995). In dieser Gestaltungsform dominiert eindeutig die Dachorganisation. Sie delegiert Aufgaben an die dezentralen Einheiten und stellt dafür die entsprechenden Ressourcen zur Verfügung. Eine moderne Form der Konzernstruktur sind Social FranchiseSysteme (BdS 2008).

Welche Struktur ein Dachverband wählt, hängt stark davon ab, was das vorrangige Selbstverständnis der Organisation ist. Young (2001) unterscheidet zielorientierte, ökonomische und politikorientierte Formen der Identität von Dachverbänden. Der Nutzen von Dachverbänden wird primär mit Unterstützungsleistungen für die Mitglieder beschrieben, weniger mit Leistungen, die unmittelbar dem Verbandszweck dienen. In einer Organisation für Behindertenbetreuung haben die regionalen und lokalen Einheiten unmittelbaren Kontakt zu den betroffenen Personen durch Betreuungseinrichtungen oder Beratungs- und Informationsleistungen. Der nationale Verband übernimmt vor allem eine Koordinations- und Kohäsionsfunktion im Sinne einer Netzwerkorganisation (Theurl 2005). Er koordiniert die Zusammenarbeit und den Austausch zwischen den Mitgliederverbänden, bietet Dienstleistungen für die Arbeit in den Mitgliederverbänden an und übernimmt die Interessensvertretung nach außen auf der (inter)nationalen Ebene. Da der Dachverband nur eine Meinung vertreten kann, müssen die Einzelansichten der Mitgliederverbände aggregiert, selektioniert und schließlich artikuliert werden. Die Komplexität dieser Aufgabe steigt mit der Heterogenität der Mitglieder. 
Grossman und Rangan (2001) benennen Governance als eines der wesentlichen Themen, in denen Management-Probleme zwischen zentralen und dezentralen Organisationen in Erscheinung treten. Young et al. (1996) sehen den Nutzen von Dachverbänden für die Governance der Mitgliederverbände vornehmlich in ihrem Beitrag zur Selbstregulierung. Dabei bieten Konzern- und föderalistische Strukturen bessere Möglichkeiten, die Mitgliederverbände zu Wirksamkeit und Verantwortlichkeit zu bringen, während Verbundstrukturen mehr zur Räsonierung der Zentrale beitragen. In einer inhaltsorientierten Analyse von 15 Non-Profit Governance-Kodizes haben von Schnurbein und Stöckli (2010) aufgezeigt, dass von Dachverbänden herausgegebene Kodizes vollständiger und umfassender sind als Lösungen von Branchen- oder Einzelorganisationen.

\section{Institutioneller Isomorphismus als theoretische Grundlage}

Wie eingangs festgestellt, hat die Bevorzugung der Prinzipal-Agenten-Theorie zu einer Konzentration der wissenschaftlichen Auseinandersetzung mit Non-Profit Governance auf den Vorstand und dessen Beziehung zur Geschäftsführung geführt. Die nachfolgende Diskussion zum Beitrag von Dachverbänden im Governance-System von Mitgliederorganisationen soll primär auf der Grundlage der Theorie des Institutionellen Isomorphismus geführt werden (DiMaggio/Powell 1983). Damit wird die Mitgliedschaft im Dachverband primär unter dem Aspekt der Verbundenheit zu einem organisationalen Feld und der daraus resultierenden Anpassungsprozesse verstanden.

Gemäß dem Institutionellen Isomorphismus gleichen sich Organisationen eines organisationalen Feldes (z. B. Produzenten gleicher Dienstleistungen) über den Zeitablauf hinweg den ähnlichen Organisationen in ihrem Umfeld durch mimetische, normative und koerzitive Prozesse an (DiMaggio/Powell 1983). Die Anpassungsprozesse dienen dabei mehr der Legitimierung als der Effizienzsteigerung. Vielmehr können Anpassungen in manchen Fällen auch die Effizienz reduzieren. Die Theorie ist eine gute Grundlage für die nachfolgende Diskussion, da der Zusammenschluss in einem Dachverband ein organisationales Feld definieren kann und aus Sicht der Mitglieder die Zugehörigkeit zum Dachverband nicht ausschließlich aufgrund von Effizienzerwägungen erfolgt.

\subsection{Mimetischer Isomorphismus}

Ist eine Organisation unsicher über die Umsetzung oder Gestaltung einer Aufgabe, sucht sie in ihrem Umfeld nach Lösungen, an denen sie sich anlehnen kann. Oftmals handelt es sich um eine andere Organisation, die als glaubwürdig erachtet wird. Ein solcher mimetischer Prozess kann z. B. die Übernahme eines erfolgreichen Prozessmanagements von einer Organisation auf die andere sein. Die Suche nach Vorbildern fördert das Denken in Benchmarks und Ratings (Drucker 1995), womit oftmals die spezifischen Eigenheiten der Organisationen übersehen werden. Im negativen Sinn können mimetische Prozesse auch zur bewussten Missachtung von allgemeingültigen Institutionen führen, wenn Regelverstöße anderer als Rechtfertigung des eigenen Handelns herangezogen werden. Oftmals laufen mimetische Prozesse nicht bewusst und aktiv gesteuert ab, sondern sind Folge unbewusster Abläufe, die sich erst im Nachhinein nachvollziehen lassen. 


\subsection{Normativer Isomorphismus}

Normativer Isomorphismus entspringt dem zunehmenden Professionalisierungsdruck in NPO (Cornforth 2003). Um diesem Trend entgegenzuwirken, werden SelfAssessment-Prozesse des Vorstandes und andere Governance-Maßnahmen ergriffen (Luoma/Goodstein 1999). Dazu zählt auch insbesondere die Vernetzung mit anderen Organisationen und in Berufsfeldern, wie sie u. a. durch Dachverbände ermöglicht wird (Meier-Schmid 2002). Ebenso sorgen Governance-Kodizes für eine Annäherung bei der Definition zentraler Inhalte der Non-Profit Governance. Governance-Kodizes werden meist von den Akteuren selbst erstellt und sind daher mehr auf Praxiserfahrung als auf wissenschaftlichen Erkenntnissen gegründet. Als Instrument der Selbstregulierung sollen sie gesetzlicher Regulierung vorbeugen. Im Gegensatz zur Entwicklung bei Wirtschaftsunternehmen hat sich im NPO-Sektor aber nicht ein einziger Governance-Kodex herausgebildet, was u. a. mit den unterschiedlichen Herausgebern der Kodizes zusammenhängt. Wie bereits erwähnt hat eine Untersuchung von 15 Kodizes aus Deutschland und der Schweiz gezeigt, dass Kodizes von Dach- oder Branchenorganisationen eine bessere Themenabdeckung haben und detailliertere Angaben zur Umsetzung machen, als Kodizes einzelner Organisationen (von Schnurbein/Stöckli 2010).

\subsection{Koerzitiver Isomorphismus}

Zuletzt geschehen Anpassungsprozesse durch Zwang bzw. Sanktionsandrohungen, die ein sehr wirkungsvolles Mittel der Einflussnahme sind (Scott 1995). Die Forderungen kommen meistens aus der näheren Umwelt des Verbandes, z. B. öffentliche Verwaltung (durch Subventionskürzungen oder Aberkennung der Steuerbefreiung), Ressourcengeber (durch Vertragskündigung) oder Dachverband (durch Ausschluss oder Vorenthalten der Zuschüsse). Besonders die Anpassung an die Dachorganisation ist bei Verbänden mit föderalistischer Struktur häufig ein Konfliktthema (DiMaggio/Powell 1983). Wissenschaftliche Untersuchungen haben gezeigt, dass Zwangsanpassungen oftmals zu Decoupling führen, d. h. erzwungene Anpassungen werden nur formal vollzogen, jedoch nicht praktisch umgesetzt (Boxenbaum/Jonsson 2008). Wenn eine Organisation sich beispielsweise zertifizieren lässt und ein Qualitätsmanagement-System einführt, heißt das nicht, dass sie auch die Vorteile und Synergien dieses Prozesses nutzt (z. B. Innovationsfähigkeit).

\section{Dachverbände als Governance-Instrument}

Der Nutzen des Dachverbandes für das Governance-System der Mitgliederorganisationen ist in der wissenschaftlichen Literatur bisher weitgehend unberücksichtigt geblieben. Mithilfe der zuvor beschriebenen isomorphen Prozesse soll dieser Mehrwert des Dachverbandes nun analysiert werden. Dabei werden Hypothesen über Wirkmechanismen von Dachverbänden als Governance-Instrument im Hinblick auf Anpassungsprozesse entwickelt. Den Annahmen des Institutionellen Isomorphismus zufolge sind Anpassungsprozesse eine Reaktion auf Unsicherheit im Umfeld oder in Bezug auf die Umsetzung. Ebenso nehmen Anpassungsprozesse zu, je eindeutiger ein organisationales Feld definiert ist (DiMaggio/Powell 1983). In der weiteren Diskussion zur Bedeutung des Dachverbandes als Governance-Instrument werden diese An-

zfwu 13/1 (2012), 50-61 
nahmen durch die Kriterien der Autonomie der Mitgliederorganisationen und der Stabilität des Umfeldes abgebildet.

Eine hohe Autonomie der Mitglieder besteht primär in Verbundstrukturen, in welchen die Mitglieder eine eigene Identität entwickeln können und eigene strategische Ziele verfolgen. In diesen Fällen ist meist der Dachverband von den finanziellen Beiträgen der Mitglieder abhängig. Eine tiefe Autonomie findet sich in föderalistischen Strukturen und in Konzernstrukturen, in denen die Dachorganisation strategische Vorgaben zur Umsetzung in den Untereinheiten entwickelt und der Mittelfluss zentral verhandelt oder gesteuert wird (Young et al. 1996). Grossman und Rangan (2001) zeigen jedoch auf, dass der kausale Zusammenhang von Autonomie und Einbindung nicht immer gegeben ist. Für sie wird Autonomie von der Frage nach der lokalen Verbundenheit, insbesondere der Fähigkeit, eine lokale Finanzierung zu gewährleisten, sowie der Frage nach der Stärke der Kundenanpassung von Leistungen getrieben. Die Einbindung in die Dachorganisation wird dagegen beeinflusst durch den daraus entstehenden Nutzen für die Mitgliederorganisation. Im Folgenden wird der Autonomiestatus als Kriterium zur Beschreibung des Einflusses von Dachverbänden auf die Governance-Strukturen einer Organisation verwandt. Ebenso wie die Autonomie der Organisation beeinflusst das Umfeld die Entwicklung der Organisation. Bradshaw (2010) verdeutlicht anhand einer Literaturanalyse zur Kontingenztheorie, dass die Gestaltung des Governance-Systems von Umfeldeinflüssen abhängig ist. Stabilität im Umfeld ergibt sich einerseits aus der „Marktsituation“, d. h. der Zusammensetzung, der Agilität und der Konsolidierung der Branche, in der die Organisation aktiv ist. Zum anderen legt das sozio-politische Umfeld die Grundlage für die Planungssicherheit und die Partizipationsmöglichkeiten (Schwarz 2005). Steht beispielsweise eine tiefgreifende Reform der Subventionspraxis an, dann führt dies unmittelbar zu Verunsicherung bei den betroffenen Organisationen.

\begin{tabular}{|c|c|c|c|}
\hline & \multicolumn{2}{|c|}{ Umfeldsituation } \\
\hline & & Unsicher & Stabil \\
\hline \multirow{2}{*}{$\begin{array}{l}\text { Autonomiegrad } \\
\text { der Mitglieder }\end{array}$} & Hoch & $\begin{array}{l}\text { Dachverband entwickelt } \\
\text { allgemeingültige } \\
\text { Institutionen }\end{array}$ & $\begin{array}{c}\text { Dachverband koordi- } \\
\text { niert mimetische Anpas- } \\
\text { sungsprozesse }\end{array}$ \\
\hline & Tief & $\begin{array}{c}\text { Dachverband als Treiber } \\
\text { des normativen Iso- } \\
\text { morphismus }\end{array}$ & $\rightarrow \begin{array}{c}\text { Dachverband sichert } \\
\text { Zugang zu spezifischen } \\
\text { Ressourcen mit Hilfe } \\
\text { von Zwang }\end{array}$ \\
\hline
\end{tabular}

Tabelle 2: Dachverbände als Governance-Instrument im Kontext von Autonomie und Umfeld sowie idealtypische Entwicklungspfade (eigene Darstellung) 
In Tabelle 2 wird eine Vier-Felder-Matrix mit Hilfe der beiden erwähnten Kriterien entwickelt, die idealtypische Konstellationen abbildet. In jedem der vier Felder laufen unterschiedliche isomorphe Prozesse ab, mit denen der Dachverband einen Einfluss auf das Governance-System der Mitgliederorganisationen ausüben kann. Die Entwicklungspfade zeigen an, wie idealtypisch Mutationen über die Feldergrenzen hinweg ablaufen sollen.

\section{(1) Unsicheres Umfeld - hohe Autonomie}

Dachverbände hoch autonomer Mitglieder in einem unsicheren Umfeld tragen insbesondere durch die Entwicklung und Durchsetzung von Standards zum GovernanceSystem der Mitgliederorganisationen bei. Die passende Organisation für solche Dachverbände sind Verbundstrukturen, denen jedoch die unmittelbare Durchsetzungsgewalt fehlt. Durch die Entwicklung und Verbreitung der Governance-Kodizes oder Best-Practice-Richtlinien entwickelt der Dachverband allgemeingültige Standards und leistet damit einen Beitrag zur Stabilisierung des Umfeldes der Mitgliederorganisationen, denn sie können sich in der Gestaltung ihrer Governance auf dieses Regelwerk berufen bzw. daran orientieren. Gleichzeitig kann eine steigende Umsetzung der unverbindlichen Richtlinien zu einer Hegemonisierung und damit zu einem Verlust an Autonomie der Mitgliederorganisationen führen, sofern auch Unsicherheit unter den Mitgliederorganisationen hinsichtlich der Definition des organisationalen Feldes und damit der eigenen Zugehörigkeit besteht. Diese Form von normativen und daraus folgenden mimetischen Anpassungsprozessen ist gerade bei sich entwickelnden oder im Umbruch befindenden Branchen von großer Bedeutung, da hier oftmals Erfahrungswerte und Orientierungshilfen fehlen.

Daraus lassen sich folgende Hypothesen ableiten:

H1: Die Mitgliedschaft in einem Dachverband trägt bei stark autonomen Organisationen in einem unsicheren Umfeld durch normative Isomorphismen zur Steigerung der Umfeldsicherheit bei.

H2: Die Mitgliedschaft in einem Dachverband fübrt bei stark autonomen Organisationen in einem unsicheren Umfeld durch normative und mimetische Isomorphismen zu Autonomieverlusten, sofern die Branchendefinition unzureichend ist.

\section{(2) Unsicheres Umfeld - tiefe Autonomie}

Die idealtypische Organisation eines Dachverbandes in diesem Feld der Matrix ist eine Konzernstruktur, die von einer weitreichenden Abhängigkeit der Mitgliederorganisationen gekennzeichnet ist. Die Unsicherheit des Umfelds verstärkt diese Abhängigkeit, da dadurch die Suche nach Alternativen zum Dachverband erschwert wird. Aufgrund dieser Konstellation fördert der Dachverband normative Isomorphismen, z. B. die Entwicklung von Branchenleitbildern oder Qualifikationen für ein bestimmtes Berufsfeld. Der Übergang zum koerzitiven Isomorphismus kann dabei jedoch fließend sein. Zur Überwindung der Unsicherheit im Umfeld wird mit der Mitgliedschaft im Dachverband ein Reputationsgewinn angestrebt. In diesem Fall ersetzt Reputation explizites Wissen und erleichtert damit Transaktionen zwischen Marktteilnehmern (Morten 1998). Grossman und Rangan (2001) sehen in der Einbindung in einen Dachverband den Vorteil, dass die Arbeit der Mitgliederorganisationen eine Wertsteigerung erfährt.

zfwu 13/1 (2012), 50-61 
Dies geschieht einerseits durch Unterstützungsleistungen der Zentrale und andererseits durch deren Beitrag zur Aufsicht der Organisationen. Besteht die Konstellation aus tiefer Autonomie und unsicherem Umfeld über einen längeren Zeitraum, führt dies früher oder später zur vollständigen Eingliederung der Mitgliederorganisationen in den Dachverband.

Daraus lässt sich folgende Hypothese ableiten:

H3: Die Mitgliedschaft in einem Dachverband trägt bei niedrig autonomen Organisationen in einem unsicheren Umfeld durch normative und koerzitive Isomorphismen zur Steigerung der Umfeldsicherheit bei.

\section{(3) Stabiles Umfeld - hohe Autonomie}

Stark autonome Mitglieder in einem stabilen Umfeld werden den Dachverband vornehmlich als Dienstleister verstehen, der Aufgaben übernimmt, die Synergieeffekte für die Mitglieder bringen. Der Dachverband ist meist geprägt von föderalistischen oder Verbundstrukturen. Die Sicherheit im Umfeld reduziert das Bedürfnis der Mitgliederorganisationen, vom Dachverband zentral gesteuerte Lösungen anzuwenden. Anpassungen im Governance-System erfolgen daher meist in mimetischen Prozessen, da der Dachverband nicht in der Position ist, allgemeingültige Normen aufzustellen oder sogar Zwang anzuwenden. Anpassungsprozesse laufen daher nur langsam ab oder in Homogenisierungsschüben, die im Allgemeinen aber kaum zu einer stärkeren Position des Dachverbandes führen.

Daraus lässt sich folgende Hypothese ableiten:

H4: Die Mitgliedschaft in einem Dachverband fordert bei hoch autonomen Organisationen in einem stabilen Umfeld mimetische Isomorphismen.

\section{(4) Stabiles Umfeld - tiefe Autonomie}

Die Mitgliedschaft in einem Dachverband in diesem Feld ergibt sich vor allem aus dessen Machtstellung aufgrund der Bedeutung spezifischer Ressourcen (z. B. Ausbildungsmonopol, zentrale Mittelvergabe). Der meist in föderalistischen Strukturen organisierte Dachverband wirkt in die Governance der Mitgliederorganisationen vor allem durch Anpassungsprozesse unter Zwang ein, deren Zielsetzung eine Homogenisierung der Mitgliederorganisationen ist. Die Vorenthaltung des Ressourcenzugangs ermöglicht eine glaubwürdige Androhung von Sanktionen und eine entsprechende Durchsetzung der Forderungen (Boxenbaum/Jonsson 2008). Verliert der Dachverband aber seine Position als Ressourcengarant, werden Mitgliederorganisationen versuchen, ihre Autonomie zu stärken.

Daraus lässt sich folgende Hypothese ableiten:

H5: Die Mitgliedschaft in einem Dachverband verliert bei niedrig autonomen Organisationen in einem stabilen Umfeld an Bedeutung, wenn Sanktionsmöglichkeiten für koerzitive Isomorphismen feblen. 


\section{Schlussbemerkung}

Ausgehend von der Feststellung, dass die Literatur zu Non-Profit Governance stark auf den Vorstand fokussiert ist, wurde in diesem Beitrag der Versuch unternommen, ein Verständnis über den Dachverband als Governance-Instrument zu entwickeln. Gerade die hohe Belastung des Vorstandes als Governance-Gremium kann durch die Beteiligung weiterer Stakeholder reduziert werden, wodurch Fehlentwicklungen früher erkannt werden können (Bradshaw 2010).

Den theoretischen Rahmen der Diskussion bildete der Institutionelle Isomorphismus. Es wurde dargelegt, in welcher Weise die Mitgliedschaft in einem Dachverband Anpassungsprozesse in den Mitgliederorganisationen fördern können. Unter Berücksichtigung der Mitgliederautonomie und der Umfeldsicherheit wurden idealtypische Ausprägungen beschrieben und Hypothesen zu Entwicklungspfaden gebildet, um die Wirkmechanismen zu verdeutlichen. Diese Hypothesen müssen in einem weiteren Schritt überprüft und gegebenenfalls angepasst werden. Weitere Forschungsanstrengungen sind zudem in Bezug auf die Berücksichtigung anderer Stakeholder von NPO zu unternehmen. Der Institutionelle Isomorphismus bietet dazu eine sinnvolle Fundierung, der mehr Beachtung geschenkt werden solle (Verbruggen et al. 2011). Ebenso lassen sich Ansatzpunkte für eine vertiefte wirtschaftsethische Auseinandersetzung formulieren. So bedarf es weiterer Überlegungen, inwiefern Autonomieverluste der Mitgliederorganisationen zu Zielkonflikten führen, wenn dadurch die Ausrichtung an den Interessen der eigenen Mitglieder unterlaufen wird. Auch stellt sich die Frage, ob Partizipation in einem Dachverband erzwungen werden kann. Schließlich können Dachverbände ihre eigene Legitimation nur dann aufrechterhalten, wenn sich die Mitgliederorganisationen aktiv beteiligen.

Obwohl es sich bei diesem Beitrag um eine theoretische Diskussion ohne empirische Überprüfung handelt, lassen sich auch Schlüsse für die Praxis ableiten. Erstens sollten sich Verbandsvertreter über die verschiedenen Formen von Anpassungsprozessen bewusst sein und Konsequenzen solcher Abläufe frühzeitig prüfen. Zweitens sollte die Mitgliedschaft in einem Dachverband nicht nur aufgrund des Nutzens für die eigene Leistungserstellung bewertet werden, sondern auch Aspekte der Umfeldunsicherheit und der eigenen Autonomie mit ins Kalkül gezogen werden. Letztlich muss auch die Mitgliedschaft im Dachverband meistens aus den Beiträgen der Mitglieder finanziert werden.

\section{Literaturverzeichnis}

Balser, D./ McClusky, J. (2005): Managing Stakeholder Relationships and Nonprofit Organization Effectiveness, in: Nonprofit Management \& Leadership, Vol. 15/No. 3, 295-315.

Boxenbaum, E./ Jonsson, S. (2008): Isomorphism, Diffusion, and Decoupling, in: Greenwood, R./ Oliver, C./ Suddaby, R./ Sahlin-Andersson, K. (Eds.): The Sage Handbook of Organizational Institutionalism, Thousand Oaks: Sage, 78-98.

Bradshaw, P. (2007): The Dynamics of Nested Governance in Nonprofit Organizations. Paper presented at Networks, Stakeholders, and Nonprofit Organization Governance: Whither (Wither) Boards? Conference, Kansas City, Mo., Apr. 26, 2007.

Bradshaw, P. (2010): A Contingency Approach to Nonprofit Governance, in: Nonprofit Management \& Leadership, Vol. 20/No. 1, 61-81. 
Brown, W. A./ Iverson, J. O. (2004): Exploring Strategy and Board Structure in Nonprofit Organizations, in: Nonprofit and Voluntary Sector Quarterly, Vol. 33/No. 3, 377-400.

Bundesverband Deutscher Stiftungen (BdS) (Hrsg.) (2008): Social Franchising, Berlin: Bundesverband Deutscher Stiftungen.

Cornforth, C. (Eds.) (2003): The Governance of Public and Nonprofit Organisations: What Do Boards Do? London: Routledge.

Cornforth, C. (2011): Nonprofit Governance Research: Limitations of the Focus on Boards and Suggestions for New Directions, in: Nonprofit and Voluntary Sector Quarterly, available online (http://nvs.sagepub.com/content/early/2011/11/14/0899764011427959. abstract; 21.03.2012).

DiMaggio, P. J./ Powell, W. W. (1983): The Iron Cage Revisited: Institutional Isomorphism and Collective Rationality in Organizational Fields, in: American Sociological Review, Vol. 48/No. 2, 147-160.

Drucker, P. F. (1995): Managing in a Time of Great Change, New York: Truman Tally Books/Plume.

Graf, A. (2007): Erfolg von Wirtschaftsverbänden: Am Beispiel der Schweiz, Wiesbaden: Gabler.

Grossman, A./ Rangan, V.K. (2001): Managing Multisite Nonprofits, in: Nonprofit Management \& Leadership, Vol. 11/No. 3, 321-337.

Luoma, P./ Goodstein, J. (1999): Stakeholders and Corporate boards: Institutional Influences on Board Composition and Structure, in: Academy of Management Journal, Vol. 42/No. 5, 553-563.

Meier-Schmid, B. (2002): Mitglieder-Marketing: Priorität Nr. 1, in: Verbands-Management, Jg. 28/Heft 2, 40-43.

Morten, T. (1998): Narrating Corporate Reputation, in: International Studies of Management \& Organization, Vol. 28/No. 3, 120-137.

Oster, S. (1995): Strategic Management of Nonprofit Organizations, New York: Oxford University Press.

Saidel, J. R. (1998): Expanding the Governance Construct: Functions and Contributions of Nonprofit Advisory Groups, in: Nonprofit and Voluntary Sector Quarterly, Vol. 27/No. 4, 421-436.

Schwary, P. (2005): Organisation in Nonprofit-Organisationen, Bern/Stuttgart/Wien: Haupt.

Scott, W. R. (1995): Institutions and Organizations, Thousand Oaks: Sage.

Theurl, T. (2005): Kooperative Governance-Strukturen, in: Arbeitspapiere des Instituts für Genossenschaftswesen der Westfälischen Wilhelms-Universität Münster, Nr. 48, Münster.

Verbruggen, S./ Christiaens, J./ Milis, K. (2011): Can Resource Dependence and Coercive Isomorphism Explain Nonprofit Organizations' Compliance With Reporting Standards?, in: Nonprofit and Voluntary Sector Quarterly, Vol. 40/No. 1, 5-32.

von Schnurbein, G. (2008): Nonprofit Governance in Verbänden, Bern/Stuttgart/Wien: Haupt.

von Schnurbein, G. (2009): Patterns of Governance Structures in Trade Associations and Unions, in: Nonprofit Management \& Leadership, Vol. 20/No. 1, 97-115.

von Schnurbein, G./ Stöckli, S. (2010): Die Gestaltung von Nonprofit Governance Kodizes in Deutschland und der Schweiz - eine komparative Inhaltsanalyse, in: Die Betriebswirtschaft, Jg. 70/Heft 6, 493-509. 
Young, D. R. (2001): Organizational Identity in Nonprofit Organizations: Strategic and Structural Implications, in: Nonprofit Management \& Leadership, Vol. 12/No. 2, 139-157.

Young, D. R. (2001): Organizational Identity and the Structure of Nonprofit Umbrella Associations, in: Nonprofit Management \& Leadership, Vol. 11/No. 3, 289-304.

Young, D. R./ Bania, N./ Bailey, D. (1996): Structure and Accountability - A Study of National Nonprofit Associations, in: Nonprofit Management \& Leadership, Vol. 6/No. 4, 347-365. 